\title{
The Biophysical Modelling of Digestive Apparatus in the Human Organism
}

\author{
Janos Vincze* and Gabriella Vincze-Tiszay \\ Health Human International Enviroment Foundation, Budapest, Hungary \\ *Corresponding author: Janos Vincze, Health Human International Enviroment Foundation, Budapest, Hungary
}

\begin{tabular}{|c|c|}
\hline ARTICLE INFO & ABSTRACT \\
\hline $\begin{array}{l}\text { Received: 幽 February 17, } 2020 \\
\text { Published: 幽 February 26, } 2020\end{array}$ & $\begin{array}{l}\text { Starting from Bertalanffy's systems theory, we wrote the differential equation } \\
\text { systems of the digestive tract. We have examined the case in which maximum efficiency } \\
\text { is achieved, because then the body is in a state of equilibrium through its homeostasis. }\end{array}$ \\
\hline $\begin{array}{l}\text { Citation: Janos Vincze, Gabriella Vincze- } \\
\text { Tiszay. The Biophysical Modelling of } \\
\text { Digestive Apparatus in the Human } \\
\text { Organism. Biomed J Sci \& Tech Res 26(1)- } \\
\text { 2020. BJSTR. MS.ID.004288. }\end{array}$ & $\begin{array}{l}\text { it has many subsystems. The digestive system has a two-level control. Block diagrams } \\
\text { can be used to examine the kind of goal orientation we want to make effective because } \\
\text { this always depends on the current needs of the given organism. A block diagram of } \\
\text { the brain-controlled digestive tract in case of normal functioning was recorded. A block } \\
\text { diagram of the part directed by the hypothetical secondary brain was also recorded. } \\
\text { We note that this part is functioning also under normal circumstances, only the brain } \\
\text { activity obscures (masks) the everyday activity of the hypothetical brain. }\end{array}$ \\
\hline
\end{tabular}

Keywords: Digestive Apparatus; Biophysical Modeling; Efficiency of Digestive; Block Diagram; Hypothetical Secondary Brain

\section{Introduction}

The main requirement for the functioning of the digestive tract is that the material going into the organism should be food and not an indigestible ballast. Substances that are indigestible to the human organism get into the body with food as well, such as cellulose forming the walls of plant seeds and plant cells. The digestion process should be optimized, we are not eating continuously, but intermittently, approx. 3-4 times a day, we do not eat unlimited quantities, because the body does not require it, etc. Defecation should be made possible 1-2 times a day. So, optimization means that fluctuations around the minimum and maximum values should be characteristic of the daily digestive process. There is a whole other kind of external restriction, namely the amount of money we have to buy certain foods, and the kinds of foods we have in our environment. Another external barrier may be whether we can dedicate enough time for meals in our daily activities.The more frequent diseases of the digestive apparatus are: gastro-duodenal ulcer, gastric ulcer, gastric cancer, hepatitis, liver cirrhosis, hepatic, gallbladder, colon cancer, chronic colitis, Chron disease, irritable colon - colopathy, chronic gastritis, digestive haemorrhage [1-6].The disturbances are seldom accentuated by stress. Hyperponderability is not related only to the digestive system [7]. Women tend to gain weight during menopause - an average of 5-10 kg, with the modification of fat spread, which deposits mainly on the abdomen and hips [8]. This weight gain can be partially explained by hormonal factors [9] (the centres of appetite and satiety are in the hypothalamus, near the ones adjusting the ovaric activity); it is favoured also by the decrease of physical activity related to age, to stress [10] - personal or professional - which lead to an excessive feeding, to searching in food - and especially sweets - a compensation for frustrations.

\section{Efficiency of Digestion in Mathematical Equations}

In order to better emphasize the quantitative nature of digestion, certain conditions are formulated. We set a number of objectives in terms of performance $\left(\mathrm{P}_{15} \mathrm{P}_{2}\right.$ and $\left.\mathrm{P}_{3}\right)$, reliability $\left(\mathrm{P}_{1}\right.$ ? $\left.\mathrm{P}_{2}\right)$ and $\cos t\left(\mathrm{C}_{\mathrm{x}}, \mathrm{C}_{2}\right.$ and $\left.\mathrm{C}_{3}\right)$. All these values are in fact the upper and lower limits of the objectives in question. We assume a number of critical factors - symbolically, A, B, X, Y, Z - as independent variables, the values of which extend over a range of values. The overall efficiency of the digestive tract $-U_{T}$ - is judged based on the weighted average of the set requirements [11]. 
Suppose that the significant performance objectives (1) are:

$P_{1}$ - maximum digestibility,

$P_{2}$ - efficiency,

$P_{3}-$ minimum duration.

The reliability objectives shall be the following (2):

$R_{1}$ - frequency of indigestion,

$R_{2}$ - recovery time following indigestion.

The cost objectives shall be the following (3):

$C_{1}$ - regional preferential of eating,

$C_{2}$ - familiar preferential of eating,

$C_{3}$ - individual preferential of eating.

For the sake of simplicity, we have included only a few critical factors (4). These shall be the following:

$A$ - general external conditions,

$B$ - the maximum digestion time to be met,

$X$ - the condition of the subsystems,

$Y$ - the quality of the food,

$Z$ - the quantity of the food.

By replacing the critical factors in the terms (1), (2) and (3), we can write the following equations:

$$
\begin{aligned}
& P_{1}=f_{P 1}(A, B, X, Y, Z) \\
& P_{2}=f_{P 2}(A, B, X, Y, Z) \\
& P_{3}=f_{P 3}(A, B, X, Y, Z) \\
& R_{1}=f_{R 1}(A, B, X, Y, Z) \\
& R_{2}=f_{R 2}(A, B, X, Y, Z) \\
& C_{1}=f_{C 1}(A, B, X, Y, Z) \\
& C_{2}=f_{C 2}(A, B, X, Y, Z) \\
& C_{3}=f_{C 3}(A, B, X, Y, Z)
\end{aligned}
$$

If we now explain the $f$ function relationships and replace them with function coefficients that may be non-linear as well as the functions other variables, the equations can be written as follows:

$$
\begin{aligned}
& P_{1}=M_{P 1 A} A+M_{P 1 B} B+M_{P 1 X} X+M_{P 1 Y} Y+M_{P 1 Z} Z \\
& P_{2}=M_{P 2 A} A+M_{P 2 B} B+M_{P 2 X} X+M_{P 2 Y} Y+M_{P 2 Z} Z
\end{aligned}
$$

$$
\begin{aligned}
& P_{3}=M_{P 3 A} A+M_{P 3 B} B+M_{P 3 X} X+M_{P 3 Y} Y+M_{P 3 Z} Z \\
& R_{1}=M_{R 1 A} A+M_{R 1 B} B+M_{R 1 X} X+M_{R 1 Y} Y+M_{R 1 Z} Z \\
& R_{2}=M_{R 2 A} A+M_{R 2 B} B+M_{R 2 X} X+M_{R 2 Y} Y+M_{R 2 Z} Z \\
& C_{1}=M_{C 1 A} A+M_{C 1 B} B+M_{C 1 X} X+M_{C 1 Y} Y+M_{C 1 Z} Z \\
& C_{2}=M_{C 2 A} A+M_{C 2 B} B+M_{C 2 X} X+M_{C 2 Y} Y+M_{C 2 Z} Z \\
& C_{3}=M_{C 3 A} A+M_{C 3 B} B+M_{C 3 X} X+M_{C 3 Y} Y+M_{C 3 Z} Z
\end{aligned}
$$

The $M$ coefficients themselves can be referenced to several variables and may in fact be non-linear functions of the variable whose coefficients they are. In addition, the overall efficiency of the digestive tract $-U_{T}-$ may be a simple additive correlation:

$$
U_{T}=W_{P 1} P_{1}+W_{P 2} P_{2+} W_{P 3} P_{3}+W_{R 1} R_{1}+W_{R 2} R_{2}+W_{C 1} C_{1}+W_{C 2} C_{2}+W_{C 3} C_{3}
$$

\section{The Digestive Tract From the Point of View of Systems}

\section{Theory}

Let us examine the digestive tract from the point of view of systems theory. We have to assume that the digestive tract as a system is an integrated whole, even though it derives from various structures and subfunctions. The other starting point should be that as a system it has a certain objective, and that the balance of these can vary greatly. The processes taking place in the digestive tract aim to optimize the functions of the system as a whole according to the objective, and to achieve maximum compatibility of the digestive subsystems. The main goal is to make the whole system work, not for a subset to operate optimally. A given system (digestive tract), with its core inputs and outputs, is a subset of a larger "super system", the human organism. The requirements for different levels of subsystems are not the same, for example, think of two subsystems: the activity of the parotid gland and the pancreas. Subfunctions can enable the overall operation, and they contribute to the success of the entire digestion. Thus, the subfunctions facilitate the functioning of the digestive tract as a whole.

So, for example, the kind of food we take in affects the attitude of the subsystems and their sub-functions to this food. For example, they have a qualitatively different attitude towards liquid foods than towards solid foods, but they also have a different attitude when consuming sugary or fibrous foods. The lifetime of digestion depends on the quality of the food, the intensity of the small and large intestine sub-functions, etc. [12]. People actually plan their meals, and buy their daily food accordingly. If we look at all this at system level, it can be illustrated in the form of a block diagram (Figure 1): 


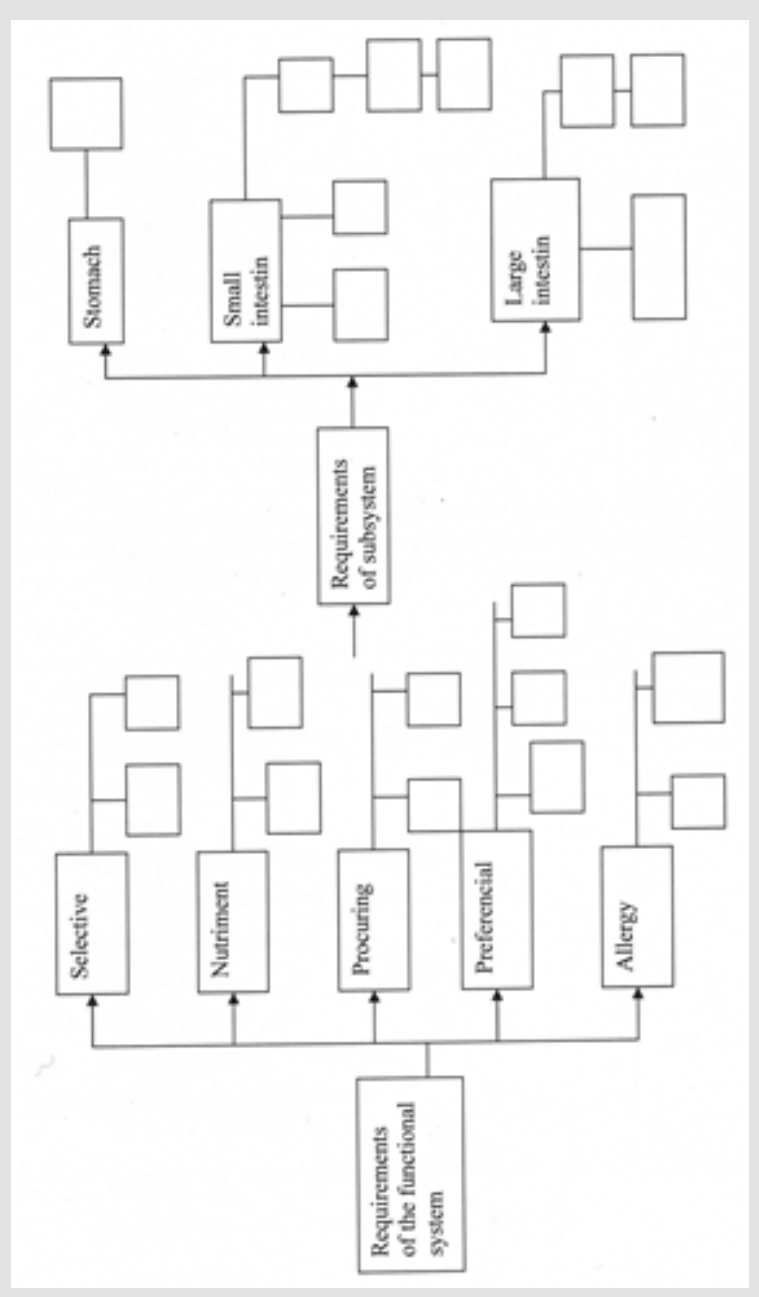

Figure 1: The block diagram of the digestive apparatus.

\section{The Two-Level Biophysical Control of the Digestive Tract in Human Organism}

The central nervous system regulation of the digestive tract is primarily achieved through the nerves in the brain. There is no center in the brain that controls digestion, and that would control all processes of the digestive tract from a single center [13]. Consider the cerebral nerves of the 12 pairs that work at different levels in the digestive tract:

a) The trigeminal nerve is mixed, with three branches: the ophthalmic, the maxillary and the mandibular branch. The first two are sensory, the third one is mixed. It innervates the facial skin and masseter muscles.

b) The facial nerve is mixed, providing the innervation of the mimic muscles, the sensitivity of taste, the function of the salivary glands from under the tongue and the jaw, and the secretory activity of the tear glands.

c) The glossopharyngeal nerve is mixed, providing the innervation of laryngeal muscle, taste perception and the salivation of the parotid. d) The vagus nerve contains mixed, sensory, motor and vegetative fibers: it controls most of the internal organs.

e) The nerve under the tongue is a motor that affects the tongue muscles.

The digestion process is described in terms of equations, symbols, circuit diagrams, as a result of which requirements can be concretely implemented in an acceptable solution (Figure 2). On the basis of systems theory, every subsystem of a system has a certain level of integrity, with its own structural and functional stability. In our opinion, this is true of the human body's digestive apparatus as the subsystems of the organism as well [14]. The concept of the subsystem purports that it must have control over its own structure. It follows, in our opinion, that digestive apparatus of the human organism contains a so-called "hypothetical secondary brain", which is responsible for certain control functions over the particular apparatus (Figure 3) [15]. The hypothetical secondary brain of the digestive apparatus is probably located in the large intestine. 


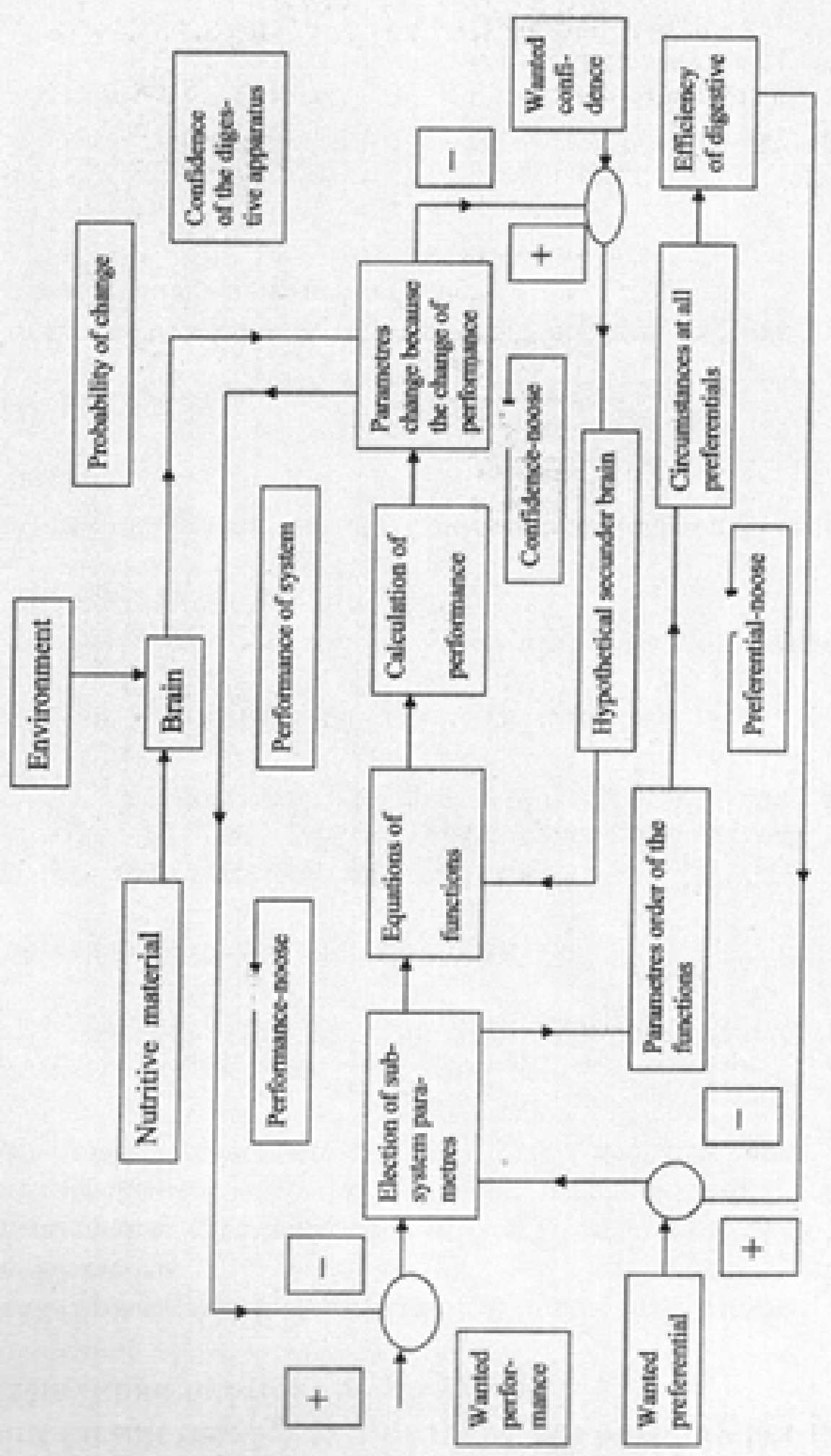

Figure 2: Process diagram of the digestive tract according to the requirements of function and structure. 


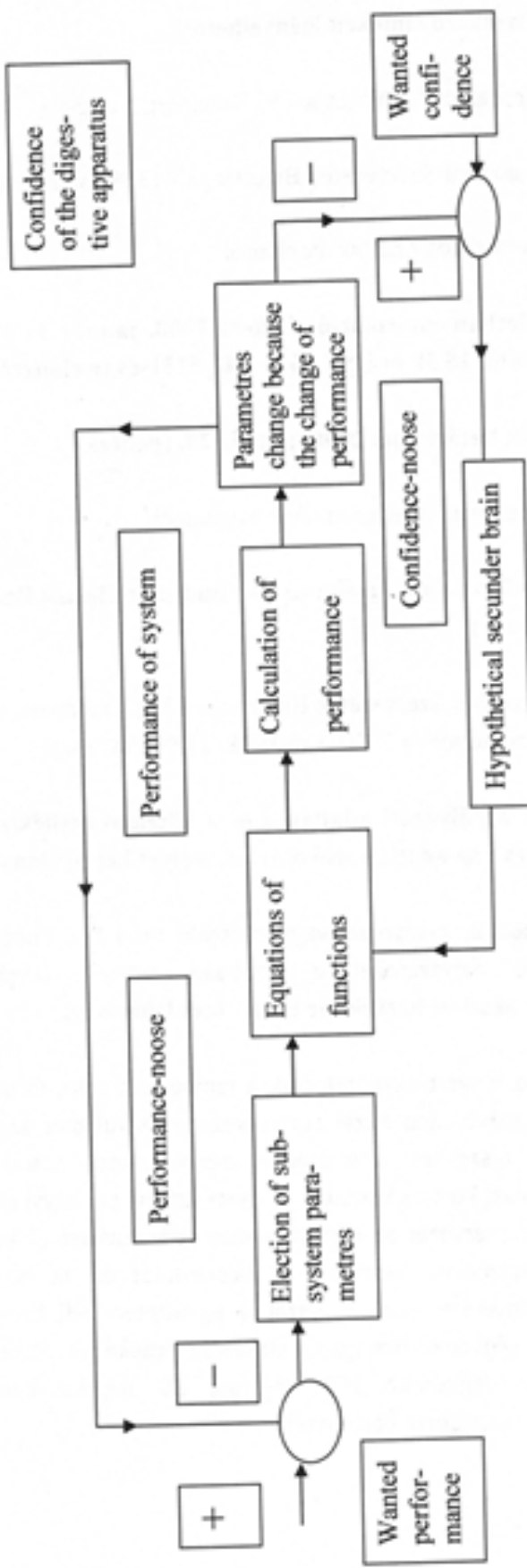

Figure 3: The block diagram of the digestive track - only "hypothetical seconder brain" -control.

\section{Conclusion}

The digestive apparatus is made of organs in which the digestion of the foods and their absorption takes place. At the same time, in the last segment of the digestive tube, the rectum, the elimination of the unabsorbed rests takes place through the act of defecation. Eating is strongly influenced by the unique past and mental state of the organism. It has never consumed certain foods (which did not exist in its environment, or did not like them); or a previous consumption of these led to illness or allergic condition. The nutrition diseases are affections caused by disturbances in the metabolism of the food substances. The metabolic deviations have multiple causes: exogenous, endogenous and mixed. The most important exogenous factor is the food intake. From the exogenous factors the most important are: the constitutional, hereditary and endocrine dysfunctions.We wrote the differential equation systems 
of the digestive tract by system theory and characterized its activity by block diagram.In our opinion, this "hypothetical secondary brains" of the digestive apparatus function continuously over a person's life, but its control function is so weak that due to the dominant activity of the central nervous system, it has not yet been detected and is still uncovered by scientific research.A fortunate model describes the given momentary acquirements but beyond this it enables assumptions which can be then translated into the language of the experiment and on this basis you can arrange certain new experimental conditions and situations. An adequately selected model will promote not only raising the question but also the scientific investigation of the phenomena. Forming a biophysical model [16] is not the task of the biologist or physicist alone, a good model can successfully construct only by common, collective work. This is typically the task of the biophysicist and a problem which falls within the competence of this discipline. Model is always an approximation; the user of the model has to take into consideration that he can approach only the absolute truth just through the endless series of relative truths.

\section{Disclosures/Conflict of interest}

The authors declare that there is no conflict of interest.

\section{References}

1. Lin JT (2014) Screening of gastric cancer, who, when, and how. Clin Gastroenterol Hepatol 12: 135-138.

2. Bernal W, Wendon J (2013) Acute liver failure. N Engl J Med 369: 25252534.

\section{ISSN: 2574-1241}

DOI: $10.26717 /$ BJSTR.2020.26.004288

Janos Vincze. Biomed J Sci \& Tech Res

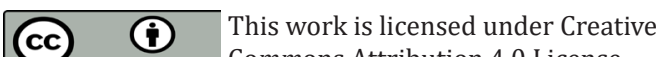

Submission Link: https://biomedres.us/submit-manuscript.php
3. Lazaridis KN, La Russo NF (2015) Primary sclerosing cholangitis. Lancet 386: 1565-1568.

4. Louvet A, Mathurin P (2015) Alcoholic liver disease: Mechanisms oninjury and targeted treatment. Nat Rev Gastroenterol Hepatol 12: 231-240.

5. Hart PA, Zen Y, Chari ST (2015) Recent advances in autoimmune pancreatitis. Gastroenterology 149: 39-51.

6. Graham C, Mullen A, Whelan K (2016) Obesity and gastrointestinal microbiota: A rewiev of associations and mechanism. Nutr Rev 73: 376382.

7. Roberts DL, Dive C, Renehan AC (2010) Biological mechanisms linking obesity and cancer risk: New perspectives. AnnuRev Med 61: 301-307.

8. Wadden TA, Sternberg JA, Letizia KA (1989)Treatment of obesity by very low calorie diet, behavior therapy, and their combination: a five-year perspective. Int J Obs 13: 39-45.

9. Casals Casals C, Desvergene B (2011) Endocrine disruptors: From endocrine to metabolic disruption. Annu RevPhys 73: 135-141.

10. Starcke K, Brand M (2016) Effects of Stress on Decision Underuncertainly. Pschilogical Bull 1323: 151-181.

11.Vincze J (2015) Biophysics of Digestive System. NDP P, Budapest, Hungary.

12. Vincze J (2007) Interdisciplinarity, NDP P, Budapest, Hungary.

13. Vincze J (2018) Medical Biophysics. NDP P, Budapest, Hungary.

14.Vincze J (2020) The Biophysical Modeling of the Evaluation of the Laboratorial Diagnosis Zones. American Journal of Internal Medicine 8(1): 1-7.

15. Vincze J (2019) The hypothetical secondary brain, Medical Research Archives 7(7): 2-3.

16. Paumgatner GM (2010) Biliary physiology and disease reflections of a physician-scientist. Hepatology 51: 1095-1110.

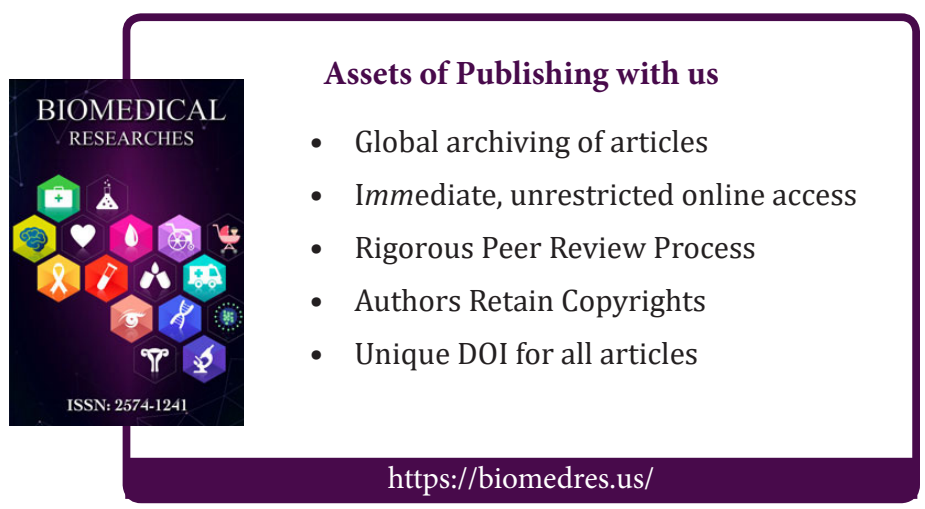

